\title{
Caracterización sociosanitaria de las familias de la localidad de Huánuco
}

\author{
Social-healthcare characterization of families in the town of Huánuco
}

Mely Ruiz-Aquino 1 1,a

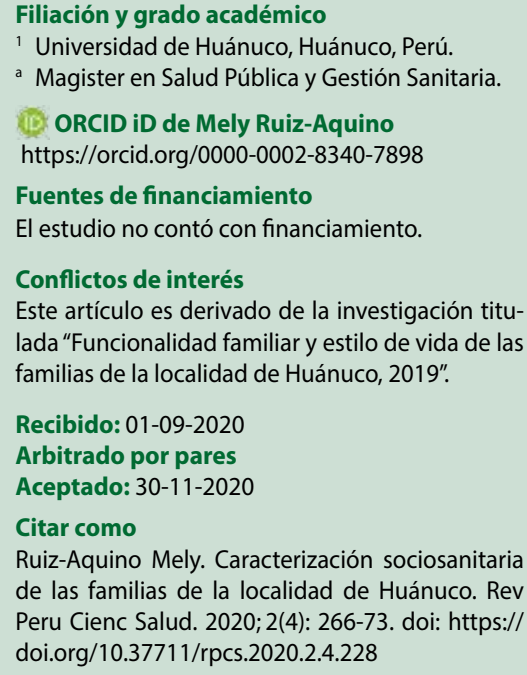

Recibido: 01-09-2020

Arbitrado por pares

Aceptado: 30-11-2020

Citar como

Ruiz-Aquino Mely. Caracterización sociosanitaria de las familias de la localidad de Huánuco. Rev Peru Cienc Salud. 2020; 2(4): 266-73. doi: https:// doi.org/10.37711/rpcs.2020.2.4.228

\section{RESUMEN}

Objetivo. Describir las características sociosanitarias de las familias de la localidad de Huánuco. Métodos. Fue un estudio con enfoque cuantitativo, de tipo descriptivo y de diseño transversal. La población fueron 1555 familias, siendo la muestra 373 , seleccionadas probabilísticamente por estratos. Se aplicó una guía de entrevista de las características generales y de las tipologías de los estilos de vida. Se usaron las medidas de frecuencias y proporciones. Resultados. Analizando las características demográficas de las familias, se halló en mayor proporción [56,8 \% (212)] que el padre es quien asume el rol de jefe de familia. El 27,9\% (104) tenía entre 40 y 49 años. Un 37,3 \% (139) declaró ser casados; un 59,5\% (222) desempeñaban tareas de manejo de maquinarias y equipos; y un $28,2 \%$ (105) tenían secundaria completa; un $70,8 \%$ (264) eran católicos y $14,2 \%$ (53) eran beneficiarios de algún programa social del gobierno. El tipo de familia, en un 57,1 \% (213), eran familias nucleares. Al describir las características del estado de salud, el 80,2 \% (299) no presentaba ningún problema de salud. El lugar donde acudían cuando se enferman [(44 \% (164)] era, en mayor proporción, el hospital. Respecto a la tipología del estilo de vida de las familias, el 34,9\% (130) tuvo un estilo de vida formalista. Conclusiones. Predominan las familias patriarcales y nucleares, con adultos medios como los jefes de familias, de condición casados, con secundaria completa, católicos y, en una gran proporción, no presentan ningún problema de salud. La tipología de estilo de vida predominante fue la formal.

Palabras clave: familias; características demográficas; características sociales; características familiares; salud familiar; estilo de vida familiar.(Fuente: DeCS BIREME)

\section{ABSTRACT}

Objective. To describe the social-healthcare characteristics of the families in the town of Huánuco. Methods. It was a study with a quantitative approach, a descriptive type and a transversal design. The population was 1555 families, the sample being 373 , selected probabilistically by strata. An interview guide of the general characteristics and typologies of the lifestyles was applied. Frequency and proportion measurements were used. Results. Analyzing the demographic characteristics of families, it was found in a higher proportion [56.8\% (212)] that the father is the one who assumes the role of head of family. $27.9 \%$ (104) were between 40 and 49 years old. A total of $37.3 \%$ (139) declared that they were married; $59.5 \%$ (222) were involved in operating machinery and equipment; $28.2 \%$ (105) had completed high school; $70.8 \%$ (264) were Catholic and $14.2 \%$ (53) were beneficiaries of some government social program. The type of family, in $57.1 \%$ (213) were nuclear families. When describing the characteristics of the health status, $80.2 \%$ (299) did not present any health problems. The place where they went when they got sick [(44\% (164)] was, in greater proportion, the hospital. With respect to the type of family lifestyle, $34.9 \%$ (130) had a formalist lifestyle. Conclusions. Patriarchal and nuclear families predominate, with average adults such as heads of families, married, with full secondary education, catholics and, in a great proportion, do not present any health problems. The predominant lifestyle typology was the formal one.

Keywords: families; demographic characteristics; social characteristics; family characteristics; family health; family lifestyle. (Source: MeSH - NLM). 


\section{INTRODUCCIÓN}

La familia ha sufrido transiciones relacionadas con diversas situaciones de orden social, económico, político o cultural, entre otros, en las diferentes esferas de la sociedad; en la cual, la familia ocupa un lugar privilegiado en la formación de comportamientos relacionados a los procesos de salud-enfermedad ${ }^{(1)}$. En este contexto, la salud es un derecho fundamental y un bien público que se construye, se vive y se disfruta, en el marco cotidiano de la vida, en el día a día, para la conservación, mantenimiento y desarrollo de todos los miembros de la familia.

La Organización Panamericana de la Salud explica que la familia constituye un microambiente promotor de salud, desarrollo y bienestar. Desde una perspectiva sociosanitarias, es un contexto complejo e integrador que constituye el vínculo entre la persona y la sociedad; por lo que está sujeta a la influencia de factores socioculturales protectores y de riesgo. El proceso salud-enfermedad de las personas, familias y comunidades es objeto-sujeto del trabajo de los equipos de salud, cuyo cometido principal es el de mejorar la calidad de vida de las poblaciones que tiene a su cuidado ${ }^{(2)}$.

Tal como señala Méndez ${ }^{(3)}$, la familia "desempeña funciones relacionadas con la satisfacción de necesidades materiales, espirituales, afectivas y educativas en sus integrantes, entre las cuales se gesta la educación para un comportamiento saludable"; no obstante, existen factores de riesgo y potencialidades que determinan la interacción con el entorno y, como tal, influyen de forma positiva o negativa en la salud individual y del grupo familiar; de modo que las alteraciones o el desequilibrio de la vida familiar tiende a provocar alguna alteración del estado de salud ${ }^{(4)}$. Louro explica que la familia "es la fuente de amor, satisfacción, bienestar y apoyo para el individuo, pero también constituye fuente de insatisfacción, malestar, estrés y enfermedad. La vivencia de problemas familiares precipita respuestas de estrés"(4).

La importancia de la familia es evidente, tanto en las políticas internacionales como en las nacionales, encarnadas en la Constitución Política del Perú; específicamente para la salud, ya que se reconoce que la familia es fundamental dentro del proceso de salud-enfermedad, Por todo ello, estudiar a la familia, su caracterización sociosanitaria, es importante para la humanidad, ya que constituye un determinante social clave de la salud para fortalecer las acciones de la salud pública. A su vez, la familia es un importante potencial para afrontar los problemas de salud y mejorar las condiciones de vida de las personas; por ello, esta temática amerita mayor atención en el marco de un sistema de salud sustentado en la práctica de la medicina familiar basada en evidencia ${ }^{(4)}$.

Es innegable que la promoción de la salud y la prevención de la enfermedad son dos temas de alta prioridad en las políticas nacionales y regionales. En este contexto, los profesionales de la salud que desarrollan acciones de atención primaria de la salud (APS) deben conocer el contexto en el que trabajan, no solo relacionado a las enfermedades prevalentes, sino al tipo de personas y familias que atienden en su jurisdicción.

Esta investigación tiene la finalidad, de describir los hallazgos sobre las características demográficas, sociales, familiares, del estado de salud y las tipologías de estilos de vida de las familias, considerando que la familia ocupa un lugar fundamental en la formación de los motivos y comportamientos implicados en el proceso saludenfermedad, en las decisiones sobre el uso de servicios de salud, entre otros ${ }^{(1)}$. También, este estudio es útil para la enfermería familiar, porque aporta información específica para el cuidado de enfermería con una gran red de apoyo a la familia, que muchas veces afronta diversos factores de riesgo, como: riesgos por grupo de edad, riesgos biológico-familiares, riesgos de medio ambiente y riesgos de servicios de salud y familia ${ }^{(5)}$.

En el contexto de la salud familiar es necesario reorientar las acciones en salud, aún fuertemente marcadas por el enfoque individual, por medio de recursos y estrategias de intervención capaces de identificar e intervenir en situaciones de vulnerabilidad que afectan las familias ${ }^{(6)}$. Como profesionales de enfermería se debe gestionar el cuidado del grupo familiar, por lo que resulta útil conocer la caracterización sociosanitaria de las familias, así como de sus tipologías de estilos de vida, ya que estas son herramientas que deben ser incorporadas en la la APS para reorientar y fortalecer las intervenciones más efectivas ${ }^{(7)}$, fomentando la práctica de estilos de vida saludables y partiendo de las características de cada familia. En base a la situación descrita, el objetivo del estudio fue describir las características sociosanitaria a las familias de la localidad de Huánuco, en el año 2019.

\section{MÉTODOS}

\section{Tipo de estudio}

Fue un estudio con enfoque cuantitativo, de tipo básico, descriptivo y con diseño transversal. Este estudio se llevó a cabo durante el último trimestre del 2019.

\section{Población y muestra}

La población fueron 1555 familias de 12 juntas vecinales registradas en la Municipalidad de la ciudad de Huánuco. 
El estudio se desarrolló en la zona urbana del distrito de Huánuco, Perú. La muestra fueron 373 familias, seleccionadas probabilísticamente y por estratos. Se incluyeron a las familias que residían por más de 3 años en la zona urbana de Huánuco y que aceptaron firmar el consentimiento informado.

El 22,3\% (83) de las familias fueron de la junta vecinal Héroes Huanuqueños; 17,2 \% (64) de Vista Alegre; 11,8\% (44) de San Pedro; el 9,4\% (35) de San Sebastián; el 6,7 \% (25) de San Cristóbal; el 5,4\% (20) de la Alameda Patrocinio; el 5,1 \% (19) del Predio El Tingo; el 4,6 \% (17) del Parque Amarilis, Milagro de Fátima y San Cristóbal, respectivamente; 4,3 \% (16) San Juan y Los Carrizales, respectivamente (ver tabla 1$)$.

\section{Instrumentos de medición}

Se aplicó la técnica de la entrevista y los instrumentos fueron la guía de entrevista de las características generales de la muestra en estudio (características demográficas, sociales, familiares y del estado de salud actual) y un cuestionario de la tipología de estilo de vida de la familia de Arellano ${ }^{(8)}$. Estos instrumentos de medición fueron validados mediante la revisión de contenidos de estudios similares (validez racional) y la validez de 7 expertos y 3 jueces, quienes validaron el contenido de los instrumentos a nivel cualitativo ambos, instrumentos de medición. La aplicación de los instrumentos de recolección de datos se hizo a través de las visitas domiciliarias.

\section{Análisis estadístico}

Se realizó el análisis descriptivo usando las frecuencias y proporciones ponderadas. Para el procesamiento de los

Tabla 1. Descripción de la junta vecinal a la que pertenecen las familias de la localidad de Huánuco, 2019

\begin{tabular}{lcc}
\hline & \multicolumn{2}{c}{$\mathbf{n = 3 7 3}$} \\
\cline { 2 - 3 } Junta vecinal & $\mathbf{f i}$ & $\%$ \\
\hline San Sebastián & 35 & 9,4 \\
Parque Amarilis & 17 & 4,6 \\
Predio El Tingo & 19 & 5,1 \\
San Pedro & 44 & 11,8 \\
Vista Alegre & 64 & 17,2 \\
Milagro de Fátima & 17 & 4,6 \\
Héroes Huanuqueños & 83 & 22,3 \\
San Cristóbal & 25 & 6,7 \\
San Juan & 16 & 4,3 \\
Los Carrizales & 16 & 4,3 \\
San Francisco & 17 & 4,6 \\
Alameda Patrocinio & 20 & 5,4 \\
\hline Fuente. Guía de entrevista de las características generales de la muestra. &
\end{tabular}

Fuente. Guía de entrevista de las características generales de la muestra. datos, se utilizó el paquete estadístico Statistical Package for the Social Sciences para Windows, versión 15.0.

\section{RESULTADOS}

Los resultados muestran las características demográficas principales de las familias en estudio; entre estas se halló que, en más de la mitad [56,8 \% (212)], el padre asume el rol de jefe de familia, seguido de un 29,8 \% (111) donde la madre es la jefa de familia. En relación al grupo de edad; el 27,9\% (104) fueron adultos medios, entre 40 y 49 años, seguido del 27,3 \% (102) de adultos mayores, entre 60 y 95 años. Referente al género, una gran proporción $[65,7 \%$ (245)] eran del género masculino. Acerca de la zona de procedencia, los jefes de familia proceden en mayor proporción de la misma zona urbana de Huánuco [91,4\% (341)] (ver tabla 2).

Tabla 2. Características demográficas de los jefes de las familias de la localidad de Huánuco, 2019

\begin{tabular}{|c|c|c|}
\hline \multirow{2}{*}{ Características demográficas } & \multicolumn{2}{|c|}{$n=373$} \\
\hline & fi & $\%$ \\
\hline \multicolumn{3}{|l|}{ Jefe de familia } \\
\hline Padre & 212 & 56,8 \\
\hline Madre & 111 & 29,8 \\
\hline Abuelo (a) & 7 & 1,9 \\
\hline Hijo (a) & 30 & 8,0 \\
\hline Tío & 10 & 2,7 \\
\hline Suegro & 3 & 0,8 \\
\hline \multicolumn{3}{|l|}{ Grupo de edad } \\
\hline Adultos jóvenes (20-39) & 73 & 19,6 \\
\hline Adultos medios (40-49) & 104 & 27,9 \\
\hline Adultos maduros (50-59) & 94 & 25,2 \\
\hline Adultos mayores (60-95) & 102 & 27,3 \\
\hline \multicolumn{3}{|l|}{ Género } \\
\hline Masculino & 245 & 65,7 \\
\hline Femenino & 128 & 34,3 \\
\hline \multicolumn{3}{|l|}{ Zona de procedencia } \\
\hline Huánuco & 341 & 91,4 \\
\hline Pasco & 11 & 2,9 \\
\hline Lima & 6 & 1,6 \\
\hline Junín & 4 & 1,1 \\
\hline Ancash & 2 & 0,5 \\
\hline Ayacucho & 3 & 0,8 \\
\hline Cuzco & 2 & 0,5 \\
\hline Cajamarca & 2 & 0,5 \\
\hline Ucayali & 2 & 0,5 \\
\hline
\end{tabular}


Al analizar las características sociales de las familias en estudio, una gran proporción de los jefes de familia eran casados [37,3\% (139)]; un 59,5\% (222) pertenecen al nivel de competencia laboral 2, quienes desempeñan como tareas el manejo de maquinarias y equipos; seguido de ocupaciones en el nivel 1 (desempeño de tareas físicas o manuales simples o rutinarias) con un $20,1 \%$ (75). Respecto al grado de escolaridad, un $28,2 \%$ (105)

Tabla 3. Características sociales de los jefes de las familias de la localidad de Huánuco, 2019

\begin{tabular}{lcc}
\hline & \multicolumn{2}{c}{$\mathbf{n}=\mathbf{3 7 3}$} \\
\cline { 2 - 3 } Características sociales & $\mathbf{f i}$ & $\%$ \\
\hline Estado civil & & \\
Casado (a) & 139 & 37,3 \\
Conviviente & 121 & 32,4 \\
Viudo (a) & 34 & 9,1 \\
Divorciado (a) & 14 & 3,8 \\
Separado (a) & 27 & 7,2 \\
Madre soltera & 26 & 7,0 \\
Padre soltero & 12 & 3,2 \\
Niveles de competencias laborales & & \\
Nivel 1 (desempeño de tareas físicas o & 75 & 20,1 \\
manuales, simples o rutinarios) & & \\
Nivel 2 (desempeño de tareas como el & 222 & 59,5 \\
manejo de maquinarias y equipos) & & \\
Nivel 3 (desempeño de tareas técnicas y & 24 & 6,4 \\
prácticas complejas) & & \\
Nivel 4 (desempeño de tareas que requie- \\
ren capacidades para resolver problemas \\
complejos)
\end{tabular}

tenían secundaria completa; el 70,8 \% (264) profesaban la religión católica; y el 85,8 \% (320) no eran beneficiarios de algún programa social del gobierno (ver tabla 3 ).

Al analizar las características familiares de los jefes de las familias en estudio, respecto al tipo de familia, se halló que un $57,1 \%$ (213) de las familias eran nucleares, un $23,9 \%$ (89) de familias extendidas y una importante proporción [13,1\% (49)] de familias monoparentales. Acerca del número de miembros de la familia, el 66,5\% (248) tenía menos de 5 miembros y, en el número de hijos, el 76,4 \% (285) tenía menos de 4 hijos (ver tabla 4).

En las características del estado de salud actual de las familias en estudio, se evidenció que una gran proporción de la muestra [80,2\% (299)] no presentaba ningún problema de salud, frente a un 19,8\% (74) que tenía problemas de salud con diagnóstico médico, de las cuales en el 8,3\% (31), la mamá era quien tiene los problemas de salud, mientras que el tipo de enfermedad más común eran las endocrinas. En relación al lugar a donde acuden cuando se enferman, el $44 \%$ (164) acudía al hospital y el 37,5\% (140) acudía a un puesto o centro de salud (ver tabla 5).

Al analizar la tipología del estilo de vida de las familias en estudio, se halló que el 34,9\% (130) tenía un estilo de vida de tipo formalista; seguido de una importante proporción [28,7\% (107)] de progresistas (ver tabla 6).

Tabla 4. Características familiares de los jefes de las familias de la localidad de Huánuco, 2019

\begin{tabular}{lcc}
\hline \multirow{2}{*}{ Características familiares } & \multicolumn{2}{c}{$\mathbf{n = 3 7 3}$} \\
\cline { 2 - 3 } Tipo de familia & $\mathbf{f i}$ & \\
Nuclear & 213 & 57,1 \\
Extendida & 89 & 23,9 \\
Monoparental & 49 & 13,1 \\
Compuesta & 11 & 2,9 \\
Viven solos & 7 & 1,9 \\
Madre soltera & 2 & 0,5 \\
Solo padre y madre & 2 & 0,5 \\
Número de miembros de la familia & & \\
Menos de 5 miembros & 248 & 66,5 \\
De 6 a 10 miembros & 116 & 31,1 \\
Más de 10 miembros & 9 & 2,4 \\
Número de hijos & & 0,3 \\
Menos de 4 hijos & 285 & 76,4 \\
De 5 a 8 hijos & & 11,8 \\
Más de 8 hijos & & \\
\hline
\end{tabular}


Tabla 5. Características del estado de salud actual de las familias de la localidad de Huánuco, 2019

\begin{tabular}{|c|c|c|}
\hline \multirow{2}{*}{ Características del estado de salud actual } & \multicolumn{2}{|c|}{$\mathrm{n}=\mathbf{3 7 3}$} \\
\hline & fi & $\%$ \\
\hline \multicolumn{3}{|l|}{ Problema de salud con diagnóstico médico } \\
\hline Sí & 74 & 19,8 \\
\hline No & 299 & 80,2 \\
\hline \multicolumn{3}{|l|}{ Quién } \\
\hline Abuela & 10 & 2,7 \\
\hline Mama & 31 & 8,3 \\
\hline Suegra & 1 & 0,3 \\
\hline Hijo & 8 & 2,1 \\
\hline Hermano & 7 & 1,9 \\
\hline Esposo & 12 & 3,2 \\
\hline Padre & 4 & 1,1 \\
\hline Sobrino & 1 & 0,3 \\
\hline \multicolumn{3}{|l|}{ Tipo de enfermedad } \\
\hline Neoplasias & 6 & 1,6 \\
\hline Enfermedades endocrinas & 31 & 8,3 \\
\hline Enfermedades del sistema cardiocirculatorio & 10 & 2,7 \\
\hline Desórdenes mentales & 8 & 2,1 \\
\hline Enfermedades del sistema nervioso central & 2 & 0,5 \\
\hline Enfermedades del sistema respiratorio & 6 & 1,6 \\
\hline Enfermedades del aparato locomotor & 5 & 1,3 \\
\hline Enfermedades del aparato genitourinario & 1 & 0,3 \\
\hline Enfermedades congénitas & 3 & 0,8 \\
\hline Enfermedades del sistema digestivo & 2 & 0,5 \\
\hline \multicolumn{3}{|l|}{ Lugar a donde acuden cuando se enferma } \\
\hline Hospital & 164 & 44,0 \\
\hline Puesto o centro de salud & 140 & 37,5 \\
\hline Curandero & 2 & 0,5 \\
\hline Consultorio o clínica particular & 26 & 7,0 \\
\hline Farmacia o botica & 28 & 7,5 \\
\hline Naturista & 10 & 2,7 \\
\hline Sanidad del policía nacional del Perú & 2 & 0,5 \\
\hline Ninguno & 1 & 0,3 \\
\hline
\end{tabular}

\section{DISCUSIÓN}

La salud familiar ha constituido una preocupación para todos aquellos que trabajan con la familia en el contexto de la APS ${ }^{(9)}$, pues es el principal potencial de promoción en salud y prevención de la enfermedad y cumple una función cuidadora y protectora de sus miembros, sobre todo aquellos que son vulnerables, enfermos y dependientes ${ }^{(10)}$.
Tabla 6. Tipología del estilo de vida de las familias de la localidad de Huánuco, 2019

\begin{tabular}{lcc}
\hline \multirow{2}{*}{ Tipo de estilo de vida } & \multicolumn{2}{c}{$\mathbf{n = 3 7 3}$} \\
\cline { 2 - 3 } & $\mathbf{f i}$ & $\%$ \\
\hline Sofisticados & 25 & 6,7 \\
Modernos & 59 & 15,8 \\
Progresistas & 107 & 28,7 \\
Formalistas & 130 & 34,9 \\
Conservadores & 52 & 13,9 \\
\hline
\end{tabular}

La familia, el entorno más cercano de las personas, no ha sido estudiada en profundidad como posible agente responsable de la salud o la enfermedad de sus integrantes, ni aun como unidad de abordaje terapéutico ${ }^{(11)}$. Por ello, el estudio sobre la vigilancia en salud y monitoreo de las condiciones de vida de las familias de un determinado territorio ${ }^{(6)}$ es una de las importantes tareas que amerita mayor atención.

La realización de este estudio se basó en el Modelo de atención integral en salud basado en familia y comunidad del Ministerio de Salud (MINSA) del Perú (2), con enfoque familiar, que reemplazó al modelo tradicional biomédico por resultar insuficiente para enfrentar las necesidades de salud de una población que experimentó cambios demográficos (envejecimiento), en el perfil epidemiológico (de enfermedades infectocontagiosas a crónicas), además de sociales y económicos.

El modelo de atención integral, centra su atención en la persona, en el contexto de su familia y la comunidad, facilitando el ejercicio pleno de sus deberes y derechos en salud, así como acercando la atención de salud a la población beneficiaria. Asimismo, se especifican los alcances de la atención integral de salud, promoviendo un modelo de gestión con enfoque territorial, con énfasis en la promoción de la salud y la prevención de la enfermedad; teniendo como eje de intervención la familia y la comunidad.

Más aún, el modelo de las determinantes sociales de la salud explica las circunstancias en que las personas viven en constante interacción, alcanzan los comportamientos y los estilos de vida, los ingresos y la posición social, la educación, el trabajo, las condiciones laborales, el acceso a servicios sanitarios adecuados y los entornos físicos que repercuten en la salud de las familias ${ }^{(12)}$. A su vez, este modelo explica la mayor parte de las inequidades sanitarias persistentes, innecesarias y evitables. En este contexto, el sistema de salud es reconocido además como indispensable para asegurar mejoras en el nivel de salud de la población ${ }^{(13)}$. 
No obstante, a partir de que la Organización Mundial de la Salud afirmó que "La salud es un hecho que determina y está determinado por el funcionamiento efectivo de la familia como unidad biosocial en el contexto de una sociedad dada", comenzó a considerarse a la familia como un determinante de la salud humana ${ }^{(14)}$.

Tal como señalan Salazar de la Cruz y Jurado ${ }^{(15)}$ en el trabajo asistencial del personal de salud, "es habitual lidiar con una amplia variedad de problemas de salud, muchos de ellos indiferenciados y a su vez con variado impacto en el individuo y su familia".

Desde el enfoque familiar, la enfermería tiene como factor común el interés por abordar el cuidado de la familia, ya que el cuidado y su gestión corresponden al ámbito del ejercicio de esta profesión ${ }^{(16)}$. En tanto el modelo teórico-metodológico de la salud familiar, explica seis dimensiones en el campo de la salud familiar: el contexto socioeconómico y cultural de vida familiar, la composición del hogar, la vivencia de procesos críticos, las relaciones intrafamiliares, el afrontamiento familiar y el apoyo social (17); las cuales fundamentan nuestros hallazgos. Bajo estas premisas, nuestros hallazgos evidenciaron que cada familia es única, con características sociosanitarias, culturales, económicos y religiosas propias que inducen a la familia a tener formas singulares y distintas de relacionarse y construir sus vínculos internos ${ }^{(18)}$.

Analizando las características demográficas de las familias, se halló que el padre es quien asume el rol de jefe de familia; vale destacar que ellos no necesariamente eran los entrevistados, pero que el entrevistado indicaba quien era el jefe de la familia. Estos resultados coinciden con el resultado de Da Silva et $a^{(6)}$, quienes hallaron familias con predominio de jefes del sexo masculino [(54,4 \% (174).] Al respecto, Sacchi et al ${ }^{(19)}$, explican que en la mayoría de los casos analizados el hombre ejerce el poder a través de la toma de decisiones y es denominado el jefe de la familia; especialmente cuando es el proveedor de los recursos económicos. A pesar de que en muchos de los casos varios miembros del hogar son los responsables de generar el ingreso familiar, cuando existe la presencia del padre, este siempre es consagrado como el principal productor. La dinámica familiar pone por lo tanto en evidencia la permanencia de familias de perfil patriarcal.

Al analizar las características familiares, el principal tipo de familia hallado fue el nuclear. Resultados similares hallaron Orozco y Velásquez ${ }^{(20)}$, quienes estudiaron 427 miembros de grupos familiares, de los cuales, el $28 \%$ fueron adultos entre 31 y 49 años; además, predominó la tipología familiar nuclear (51\%), seguida de la extensa modificada (18\%). A diferencia de nuestro estudio, en el estudio de Ávila (1), encontraron un $41 \%$ extensas y solo el $22,3 \%$ eran familias nucleares.

Al describir las características del estado de salud, una gran proporción de la muestra [80,2 \% (299)] no presentó ningún problema de salud. Similares resultados hallaron Orozco, y Velásquez ${ }^{(20)}$, quienes reportaron que la mayoría las personas que fueron estudiadas se percibieron sanas (64 \%), aunque prevalecieron en menor proporción patologías crónicas como: hipertensión arterial (53\%), cáncer (40 \%) y diabetes (39\%).

En la tipología del estilo de vida de las familias, la gran mayoría tuvieron un estilo de vida formalista. Al respecto, Arellano (21) explica que los formalistas o adaptados son hombres buscadores del respeto social menos ambiciosos y con menos aspiraciones que otros estilos de vida. No les gusta el riesgo y se interesan en la familia y en los amigos, son respetuosos de la religión y buscan la marca como garantía. Además, buscan el ahorro y educarse, son tradicionales en su consumo y aspiran a ser honrados y respetados.

Asimismo, Arellano ${ }^{(22)}$, afirma que es el estilo de vida que determina la forma de gastar, el nivel de importancia que se le brinda al dinero, la inclinación hacia el ahorro, para la toma de decisiones racionales o emocionales, para las inclinaciones de búsquedas de información, así como la necesidad de status o pertenencia mediante la compra. Urbizagástegui ${ }^{(23)}$ también explica que los estilos de vida son maneras de ser y actuar compartidas por un determinado grupo significativo de personas. Las personas formalistas se parecen por razones sociodemográficas (edades, sexo, nivel socioeconómico), psicológicas (actitud, motivaciones e intereses), conductuales, de equipamiento y de infraestructura. Son un grupo de personas que se parecen en su forma general de ser, les atraen cosas similares y creen en aspectos comunes.

La identificación de la tipología del estilo de vida familiar es importante según, García ${ }^{(11)}$ porque, la familia es el ambiente central en el cual se desarrollan, mantienen y cambian los hábitos de salud. Los familiares tienden a compartir los mismos estilos de vida, los hábitos de salud, incluyendo patrones de alimentación, de actividad física y uso de sustancias (tabaco, alcohol, drogas). Los estilos de vida de un individuo se relacionan con los de su pareja y los hábitos de salud de los padres influyen el tipo de conductas que los hijos adoptarán en el futuro.

En suma, las condiciones sociales suelen afectar a todos los miembros de la familia, ya que viven en 
el mismo lugar, generando de manera simultánea enfermedades agudas o crónicas en múltiples integrantes, por lo que se concluye indicando que, en el caso de las familias huanuqueñas, prevaleció las familias patriarcales y de tipo nuclear, siendo adultos medios los jefes de familias en gran proporción, de condición casados y con un nivel de estudios de secundaria completa y religión católica. Una gran proporción de la muestra no presenta ningún problema de salud. La tipología de estilo de vida en mayor proporción fueron la de formales.

Estos resultados brindan elementos a favor de la necesidad de intervenciones a nivel de la salud familiar, como vía para mejorar los indicadores de salud. Los resultados exigen a la enfermería y otros trabajadores de la salud iniciar acciones que aseguren una prestación de servicios de salud tendentes a disminuir riesgos y mejorar la salud familiar; de tal modo que, teniendo en cuenta la caracterización sociosanitarias de las familias, es indispensable adelantarnos a circunstancias previsibles en la vida del individuo y su familia.

Como limitaciones, vale destacar que se encontró escasa literatura acerca de la caracterización sociosanitarias de las familias; asimismo, fueron omitidas en el estudio las variables económicas. En lo que se refiere a las perspectivas futuras, sería conveniente que los profesionales de la salud realicen valoraciones más profundas de la unidad familiar y no se centren solo a las variables analizadas en este estudio, con el objeto de ampliar el horizonte del estudio, para una visión más integral; asimismo, se recomienda formular planes de atención de salud según las características sociosanitarias de las familias, propiciando estrategias para una mayor participación de las mismas.

Desde el quehacer educativo, se espera que todos los profesionales de la salud adquieran habilidades a través de metodologías vivenciales e interactivas durante su formación para trabajar con familias y contribuir de este modo a la mejora de la calidad de vida y, por supuesto, los saludables mediante la aplicación de las estrategias de promoción de la salud. Por último, se espera que este estudio represente una contribución para la salud familiar.

\section{Agradecimiento}

A las familias que dieron su consentimiento de participar en el estudio.

\section{REFERENCIAS}

1. Ávila Oliva, M. Caracterización de la familia del anciano con deterioro cognitivo. Rev Cuba Salud Pública [Internet] 2012; 38(2): 246-52. Disponible en: http://scielo.sld.cu/scielo.php?script=sci_arttext\&pi$d=$ S0864-34662012000200007

2. Ministerio de Salud. Dirección General de Salud de las Personas. Secretaría Técnica Comisión de Renovación del MAIS-BFC. Modelo de Atención Integral en Salud basado en Familia y Comunidad [Internet] [Consultado 2020 nov 15] Disponible en: https://determinantes.dge. gob.pe/archivos/1880.pdf

3. Méndez Sutil V, Louro Bernal I, Bayarre Vea H. Caracterización de la salud familiar en familias de niños con éxito escolar. Rev Cuba med gen integr [Internet] 2011; 27(1): 1-9 [Consultado 2020 agost 15] Disponible en: http://scielo.sld.cu/scielo.php?script=sci_arttext\&pi$\mathrm{d}=$ S0864-21252011000100001

4. Louro Bernal I. La familia en la determinación de la salud. Rev Cubana Salud Pública [Internet] 2003 Mar; 29(1): 48-51 [Consultado 2020 Dic 27] Disponible en: http://scielo.sld.cu/scielo.php?script=sci_arttext\&pi$\mathrm{d}=$ S0864-34662003000100007\&Ing=es.

5. Pérez Giraldo B. Caracterización de las familias con adolescentes gestantes: Perspectiva de riesgo y grado de salud familiar. Aquichan [Internet] 2003 Dic; 3(1): 21-31 [Consultado 2020 Dic 27] Disponible en: http:// www.scielo.org.co/scielo.php?script=sci_arttext\&pi$d=S 1657-59972003000100005 \&$ Ing =en

6. Da Silva Ríos T, Martins Alvarenga M, De Campos Oliveira M. Evaluación de la vulnerabilidad de familias asistidas en la Atención Básica. Rev Lat Am Enfermagem [Internet] 2012; 20(5): 9 pantallas [Consultado 2020 feb 15] Disponible en: https://www.scielo.br/pdf/rlae/v20n5/es_16.pdf

7. Herrera Santí PMH. La familia funcional y disfuncional, un indicador de salud. Rev Cuba Med Gen Integr [Internet] 1997; 13(6): 591-5 [Consultado 2020 feb 15] Disponible en: http://scielo.sld.cu/scielo.php?script=sci_arttext\&pid=S0864-21251997000600013

8. Arellano R. Los Estilos de Vida (EdV) [Internet] [Consultado 2020 Sep 19] Disponible en: https://www.arellano. pe/estilos-de-vida/

9. Martínez-Montilla JM, Amador-Marín B, Guerra-Martín MD. Estrategias de afrontamiento familiar y repercusiones en la salud familiar: Una revisión de la literatura. Enferm. glob. [Internet]. 2017; 16(47): 576604 [Consultado 2021 Ene 5] Disponible en: http:// scielo.isciii.es/scielo.php?script=sci_arttext\&pi$d=S 1695-61412017000300576 \&$ Ing =es

10. Díaz Cárdenas S, Tirado Amador LR, Madera Anaya MV. La Salud Familiar al servicio de la Odontología. Rev Clin Med Fam [Internet] 2013 Feb; 6(1): 6162 [Consultado 2021 Ene 5] Disponible en: http:// scielo.isciii.es/scielo.php?script=sci_arttext\&pid=S1699-695X2013000100013\&lng=es. http://dx.doi. org/10.4321/S1699-695X2013000100013

11. García-Huidobro D. Enfoque familiar en la Atención Primaria: Una propuesta para mejorar la salud de todos. Rev. méd. Chile [Internet] 2010 Nov; 138(11): 1463-1464 [Consultado 2021 Ene 4] Disponible en: https://scielo.conicyt.cl/scielo.php?script=sci_arttext\&pi$\mathrm{d}=$ S0034-98872010001200019\&lng=es

12. Berenguer GMC, Pérez RA, Dávila FM, et al. Determinantes sociales en la salud de la familia cubana. MediSan. 2017; 21(01): 61-73.

13. Berenguer Gouarnaluses MC, Pérez Rodríguez A, Dávila Fernández M, Sánchez Jacas I. Determinantes sociales 
en la salud de la familia cubana. MEDISAN [Internet] 2017 Ene; 21(1): 61-73 [Consultado 2020 Dic 27] Disponible en: http://scielo.sld.cu/scielo.php?script=sci_arttext\&pid=S1029-30192017000100008\&lng=es

14. Organización Mundial de la Salud. Índices estadísticos de la salud de la familia : informe de un Grupo de Estudio de la OMS [se reunió en Ginebra del 17 al 21 de febrero de 1975] [Internet] [Consultado 2020 octb 12] Disponible en: https://apps.who.int/iris/handle/10665/40938

15. Salazar de la Cruz M, Jurado-Vega A. Niveles de Intervención Familiar. Acta méd. peruana [Internet] 2013 Ene; 30(1): 37-41 [Consultado 2021 Ene 4] Disponible en: http://www.scielo.org.pe/scielo.php?script=sci_arttext\&pid=S1728-59172013000100007\&lng=es

16. Ministerio de Salud (Chile). Subsecretaría de Redes Asistenciales. Norma General Administrativa N. ${ }^{\circ} 19$ "Gestión del cuidado de enfermería para la atención cerrada", Exenta N. ${ }^{\circ} 1127$, del Ministerio de Salud. Santiago, 14 de diciembre de 2007, publicada el 25 de diciembre de 2007. [Consultado 2018 dic 4] Disponible en: http://juridico1.minsal. cl/RESOLUCION_1127_07.doc

17. Louro BI. Modelo de salud del grupo familiar. Rev Cubana Salud Pública [Internet] 2005;31(4):332-7 [citado 24 de septiembre de 2010] Disponible en: http://scielo.sld.cu/scielo.php?script=sci_arttext\&pi$d=$ S0864-34662005000400011
18. Sánchez G, Aguirre M, Solano N, Viveros E. Sobre la dinámica familiar. Revisión documental. Cultura, Educación y Sociedad. 2015, 6(2), 117-138.

19. Sacchi M, Hausberger M, Pereyra A. Percepción del proceso salud-enfermedad-atención y aspectos que influyen en la baja utilización del Sistema de Salud, en familias pobres de la ciudad de Salta. Salud Colect. 2007; 3(3): 271-283.

20. Orozco Castillo ML, Velásquez Gutiérrez VF. Caracterización del riesgo familiar en familias de estudiantes del Programa de Enfermería, de una Universidad Pública. Hacia la Promoción la Salud. 2010; 15(1): 78-91.

21. Arellano, R. Estilos de vida en el Perú [Internet] [Consultado 2020 abr 21] Disponible en: https://blogs.upc.edu. pe/blog-de-administracion-y-marketing/entorno/losestilos-de-vida-en-el-peru

22. Arellano Cueva, R. Estilos de vida en el Perú. Cómo somos y pensamos los peruanos del siglo XXI. Lima: Consumidores y Mercados; 2000.

23. Urbizagástegui Torres ES. Influencia del estilo de vida y los hábitos de consumo en la compra de productos naturales en hombres y mujeres de Lima [Tesis para optar al título profesional de licenciado en Marketing] Lima: Universidad San Ignacio de Loyola, Facultad de Ciencias Empresariales; 2017 